\title{
DOPING AND CHARACTERIZATION OF WIDE-GAP II-VI EPILAYERS*
}

\author{
G. KaRCZEWSKI AND T. WoJTowicz \\ Institute of Physics, Polish Academy of Sciences \\ Al. Lotników 32/46, 02-668 Warszawa, Poland
}

In this paper we review the properties of $n$-type doped $\mathrm{ZnSe}$ and CdTe epilayers grown by molecular beam epitaxy on (100) GaAs substrates. Recent results of photoluminescence, transport measurements, secondary ion mass spectroscopy and deep-level transient spectroscopy are discussed. A major emphasis is placed on the effect of different dopant species and the role of the deviation from stoichiometry on the doped epitaxial layers. Since deep defect states play an important role in determining the properties of the doped materials, considerable attention is directed towards characterization and identification of deep-lying defect states, both native and introduced by dopants. In particular, in the case of ZnSe the deep-level transient spectroscopy results clarify why $\mathrm{Cl}$ is superior to $\mathrm{Ga}$ as an effective $n$-type dopant. They provide strong evidence that chlorine - unlike $\mathrm{Ga}$ - does not introduce by itself any detectable deep defects into the $\mathrm{ZnSe}$ lattice. In the case of $\mathrm{CdTe}$, we focus on the influence of the deviation from stoichiometric growth conditions in the molecular beam epitaxy process and on the properties of In doped layers. We discuss resistivity, Mn diffusivity and the presence of various deep defects in layers grown at different $\mathrm{Cd} / \mathrm{Te}$ flux ratios.

PACS numbers: 68.55.Ln, 71.55.-i, 71.55.Gs

\section{Introduction}

The wide-gap II-VI materials continue to attract the attention of numerous research groups, because of their extraordinary efficiencies for photo- and electroluminescence at near-band-gap energies. These properties make them highly promising candidates for optoelectronic device applications operating in the spectral region which is not covered by the standard III-V materials (i.e., the "blue-green" spectral region). The steady flow of reports on the fabrication of blue light emitting diodes (LEDs) and optically or electron-beam pumped lasers has been crowned by the first demonstration of laser action in $\mathrm{ZnSe-based} \mathrm{injection} \mathrm{diode} \mathrm{lasers}[1,2]$.

*This work is supported by the Committee for Scientific Research through grant 8 T11B 02108. 
The major obstacle standing for many years in the way of applications of II-VI's was the inability to control the doping process - and specifically, the inability to achieve reproducible growth of low-resistivity materials of both $n$ - and p-type. Employing chlorine for donor doping [3] and active nitrogen for acceptor doping [4] has been the turning point in the evolution of the II-VI materials based devices. The new doping methods not only opened perspectives for commercial applications of these materials, but also - for the first time - made heavily doped II-VI's available for investigation of basic physical properties. In spite of the empirical success in the fabrication of the first II-VI diode devices, the understanding of the basic properties of the doped material is still fragmentary and - if not addressed systematically - may stand in the way of further technological progress. In particular, there is little known about deep states introduced into the II-VI lattices by the various doping procedures and by the different dopant species.

In this review we will focus only on two representatives of the II-VI family - $\mathrm{ZnSe}$ and $\mathrm{CdTe}-$ and on two specific aspects of the doping procedure - the choice of the dopant species and the role of stoichiometry during the molecular beam epitaxy (MBE) growth process. In order to illustrate the first subject, we will compare optical and transport properties of $n$-type $\mathrm{ZnSe}$ doped either with gallium or chlorine [5-8]. The consequences of deviations from stoichiometric growth conditions for the doped material will be shown based on investigations of CdTe films doped with indium [9-12]. All the discussed results have been obtained on samples grown by MBE on (100) GaAs substrates, either at the University of Notre Dame ( $\mathrm{ZnSe})$ or at the Institute of Physics in Warsaw (CdTe). In an effort to present information about the subject, we will review data obtained both at Notre Dame and at the Institute of Physics by photoluminescence, transport measurements, and capacitance methods. Special emphasis will be placed on deep-level transient spectroscopy (DLTS) and double correlation DLTS (DDLTS), which constitute sensitive experimental tools when used for identifying deep levels in semiconductors. The identification of defects is of primary importance for investigations of the doping processes and their consequences for the doped materials.

\section{The choice of dopant species: $\mathrm{Ga}$ versus $\mathrm{Cl}$ in $\mathrm{ZnSe}$}

One of the most frequently used impurities for $n$-type doping of $\mathrm{ZnSe}-$ and consequently the best known in this context - is gallium [5, 13,14]. This group-III element substitutes for $\mathrm{Zn}$ in the $\mathrm{ZnSe}$ lattice, acting as a shallow donor with an activation energy of about $26 \mathrm{meV}$. Its ready a vailability in high-purity elemental form, and its low chemical reactivity, makes gallium an ideal choice for most of the crystal growth methods, and especially MBE. Unfortunately the highest carrier concentrations achieved by gallium doping do not exceed the $10^{18} \mathrm{~cm}^{-3}$ level [13]. Attempts to reach higher carrier concentrations by introducing higher doses of gallium usually result in a saturation - or even a decrease - of the carrier concentration [14]. High Ga-doping is also accompanied by another effect which is harmful to optoelectronic device performance: a significant decrease in the near-band-gap photoluminescence in favor of an increase in deep-level optical transitions. The saturation of the carrier concentration and the increase in the deep-level lumines- 
cence are both believed to be caused by the so-called "self-compensation" effect, i.e., by the creation of deep acceptor-like states as a result of heavy $\mathrm{Ga}$ doping.

As an alternative to $\mathrm{Ga}$ atoms, group-VII elements substituting for Se in the $\mathrm{ZnSe}$ lattice can be employed as donors in this material $[3,15,16]$. Due to their strong ionicity, group-VII atoms are expected to be more efficient as donors than gallium. Ilere chlorine appears to be an ideal candidate for doping during MBE, because a solid source of $\mathrm{Cl}$ is available in the form of $\mathrm{ZnCl}_{2}$. This compound sublimates at temperatures slightly higher than room temperature, so that the doping level of $\mathrm{Cl}$ can easily be controlled by the temperature of the cell containing $\mathrm{ZnCl}_{2}, \mathrm{~T}_{\mathrm{Cl}}$. Chlorine doping has already been shown to be very effective: the highest carrier concentrations ever reached in $\mathrm{ZnSe}$ - exceeding $10^{19} \mathrm{~cm}^{-3}$ - have recently been reported for $\mathrm{ZnSe}: \mathrm{Cl}$ grown by MBE [15]. The high Hall mobility of $2480 \mathrm{~cm}^{2} /(\mathrm{V} \mathrm{s})$ at $40 \mathrm{~K}$ and the low compensation ratio $N_{\mathrm{A}} / N_{\mathrm{D}}=4 \%$, obtained for a film doped to a level of $3 \times 10^{17} \mathrm{~cm}^{-3}$, indicate the excellent quality of the chlorine-doped material [3].

Figure 1 provides a comparison of the photoluminescence spectra obtained on $\mathrm{ZnSe}$ epilayers doped with $\mathrm{Ga}$ and with $\mathrm{Cl}$ of nearly the same net donor concentrations on the order of $10^{17} \mathrm{~cm}^{-3}$ [8]. A typical luminescence spectrum of an undoped $\mathrm{ZnSe}$ epilayer (not shown in Fig. 1) is dominated by two distinct emission lines in the band-edge region - the neutral donor-bound exciton line (marked $I_{2}$ ) at $2.796 \mathrm{eV}$, and the free exciton line (marked $E_{x}$ ) at $2.802 \mathrm{eV}$. A much weaker bound exciton line $\left(I_{1}^{\text {deep }}\right)$ at $2.78 \mathrm{eV}$ signifies the presence of a number of deep

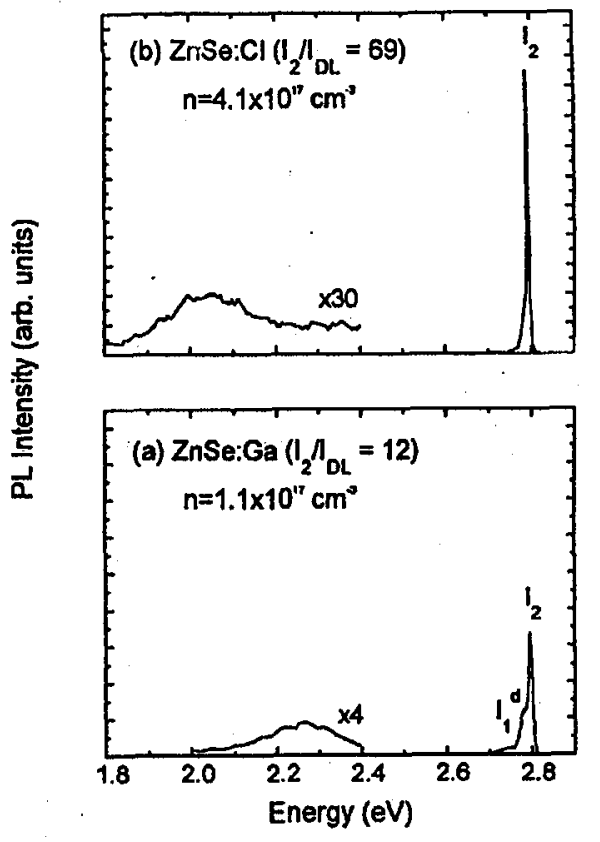

Fig. 1. Phiotoluminescence spectra of (a) gallium doped and (b) chlorine doped $\mathrm{ZnSe}$ layers. In both cases the doping level was of the same order $10^{17} \mathrm{~cm}^{-3}$ (after Ref. [8]). 
acceptors, which partially compensate the native donors which dominate the electronic properties of undoped $\mathrm{ZnSe}$. Donor doping causes a strong increase in the bound exciton line $I_{2}$ and the disappearance of the free exciton emission $E_{x}$. However, there are two major differences in photoluminescence (PL) spectra of Gaand $\mathrm{Cl}$-doped $\mathrm{ZnSe}$ layers shown in Fig. 1. First, for Ga-doped films the band of deep-level (DL) emission is much stronger, and the $I_{2}$-line is slightly weaker than for Cl-doped films. As a result, the ratio $I_{2} / I_{\mathrm{DL}}$ is about six times higher for $\mathrm{ZnSe}: \mathrm{Cl}$ than for ZnSe:Ga. Second, the DL band in $\mathrm{ZnSe}: \mathrm{Ga}$ occurs at a higher energy (around 2.2-2.3 eV) than in the case of $\mathrm{ZnSe}: \mathrm{Cl}$ (around $2.0 \mathrm{eV}$ ).

The photoluminescence data are in very good agreement with earlier studies on $\mathrm{Cl}$-doped $\mathrm{ZnSe}$ films [3]. In particular, they fully confirm the conclusion that from the point of view of the optical properties $\mathrm{Cl}$ is superior to $\mathrm{Ga}$ as an $n$-type dopant.

The superiority of $\mathrm{Cl}$ donors over Ga donors can be understood by taking into consideration DLTS results obtained from $\mathrm{ZnSe}: \mathrm{Cl}$ and $\mathrm{ZnSe}: \mathrm{Ga}$ [5-7]. In Ga-doped $\mathrm{ZnSe}$ films two dominant deep levels, labeled as $E_{\mathrm{Ga}}$ and $E_{\mathrm{Ga}}$, were detected. Figure 2a shows the concentration of traps $E_{\mathrm{Ga} 1}$ and $E_{\mathrm{Ga} 2}$ as a function of the shallow donor concentration. The concentrations of both types of traps are linearly proportional to the $\mathrm{Ga}$ donor concentration, but their ratio remains independent of this concentration. The first observation clearly indicates that both traps are related to the $\mathrm{Ga}$ dopant. The second observation suggests that both traps originate from the same defect. In other words, Ga-doping intro-

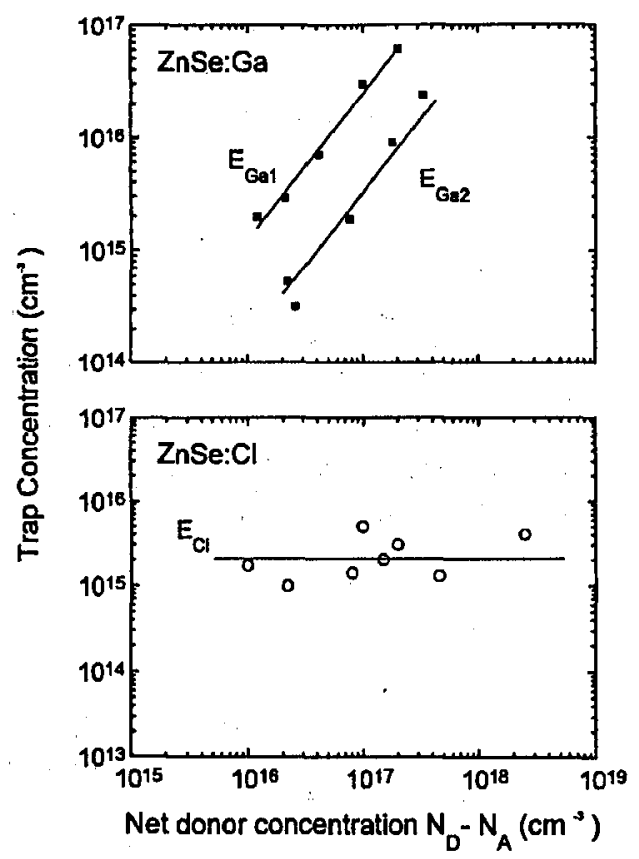

Fig. 2. Concentration of defects detected in gallium and chlorine doped $\mathrm{ZnSe}$ as a function of the net donor concentration (after Refs. [5,6]). 
duces into ZnSe a deep defect - probably a complex of substitutional gallium and zinc vacancy in the next-nearest-neighbor positions, $\mathrm{Ga}_{\mathrm{Zn}}-\mathrm{V}_{\mathrm{Zn}}$. Such a deep defect possesses two electron levels [5]. The ground level of this defect center (with the activation energy of $0.53 \mathrm{eV}$ ) is attributed to a donor-like one-electron state $(0 /+)$. The higher, two-electron level is attributed to an acceptor-like state $(-/ 0)$. The binding energy of the second electron on the defect is $0.17 \mathrm{eV}$. Because the acceptor-like state can capture a second electron, an increased concentration of such defect complexes effectively compensates the shallow donors. The configuration diagram for this defect, which is consistent with all experimental data, was proposed in Ref. [5].

In the case of $\mathrm{Cl}$-doped $\mathrm{ZnSe}$ the DLTS results look completely different. In all investigated layers only one dominant defect, labeled as $E_{\mathrm{Cl}}$, was detected. In addition, it was established that the concentration of this trap does not depend on the chlorine concentration and in all samples remains equal to approximately $2 \times 10^{15} \mathrm{~cm}^{-3}$ (see Fig. 2b). These results indicate that traps $E_{\mathrm{Cl}}$ are not introduced by the doping procedure, rather they are associated with intrinsic defects. Because chlorine doping does not cause formation of any additional defects in ZnSe layers, the Cl-doped materials exhibit much better optical and transport properties than those doped with gallium.

\section{The role of stoichiometry: In-doped CdTe}

Besides the proper choice of the dopant species, the other important factor which can strongly influence the effectiveness of the MBE doping of II-VI materials is the stoichiometry of the doped layer controlled by the metal-to-nonmetal flux ratio.

In the case of In-doped CdTe layers it was demonstrated that the free carrier concentration can be controlled in two independent ways: either by changing the flux of indium for a constant $\mathrm{Cd} / \mathrm{Te}$ flux ratio or by adjusting the $\mathrm{Cd}$ to $\mathrm{Te}$ flux ratio for a constant In flux. The effectiveness of the first method is shown in Fig. 3, where free carrier concentrations determined from the Hall effect measurements and concentrations of indium atoms measured by secondary ion mass spectroscopy (SIMS) are plotted as a function of the inverse temperature of the In-cell, $1 / T_{\text {In }}$. All the samples were grown using the same $\mathrm{Cd} / \mathrm{Te}$ beam equivalent pressure ratio $(\mathrm{Cd} / \mathrm{Te}=1.5)$. By adjusting the In-cell temperature, $T_{\mathrm{In}}$, the carrier concentration could be controlled in a wide range - from $8 \times 10^{14}$ up to $1.5 \times 10^{18} \mathrm{~cm}^{-3}$. For low In-cell temperatures (i.e. low In fluxes), the concentrations of both free carriers and In atoms were very close. This indicates that the efficiency of the In-doping process was close to $100 \%$. For higher In fluxes, when the concentration of In atoms exceeds the level of $10^{18}$, the Hall concentration significantly decreased, which points to an onset of strong compensation by self-activated acceptors $[9,10]$.

The consequence of the deviation from stoichiometry on the effectiveness of In-doping is shown in Fig. 4a. The plot presents the resistivity of a series of CdTe epilayers grown for fixed CdTe and In fluxes, but at different values of Cd/Te flux ratios. In Fig. 4a instead of carrier concentration we refer to resistivity, because for $\mathrm{CdTe}$ the resistivity depends linearly on the inverse of the carrier concentration. This simple proportionality, which holds for over six orders of magnitude [9], is a 


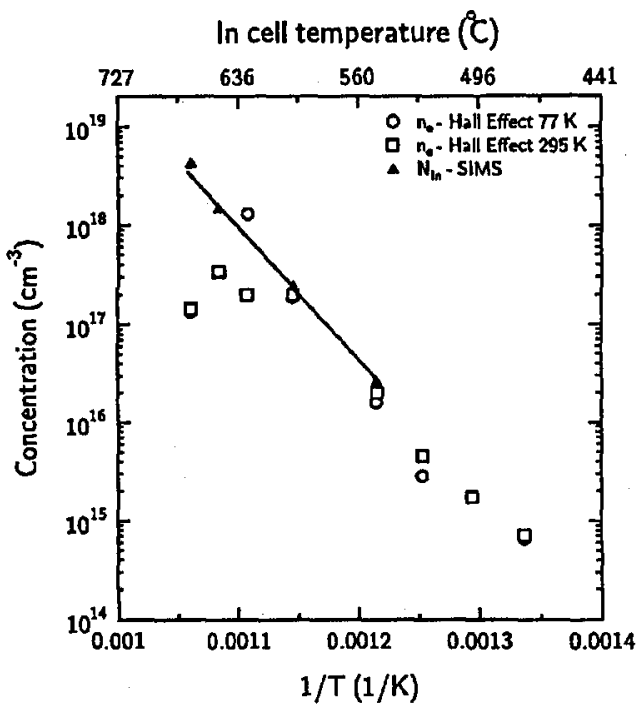

Fig. 3. Carrier concentration at 77 (circles) and $295 \mathrm{~K}$ (squares) and concentration of In atoms determined by SIMS (triangles) for In-doped CdTe films as a function of the inverse temperature of the In-cell (after Ref. [9]).

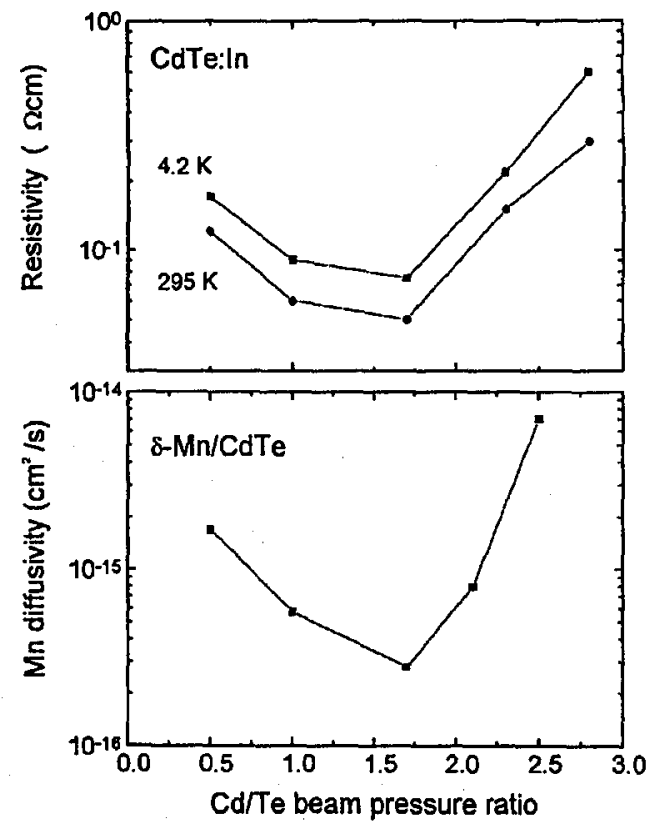

Fig. 4. Resistivity of In-doped CdTe epilayer (a) and diffusivity of Mn atoms (b) as a function of the $\mathrm{Cd} / \mathrm{Te}$ flux ratio (after Refs. $[9 ; 21]$ ). 
consequence of the experimental fact that the electron mobility in CdTe:In layers appears to be practically independent of the electron concentration and of the temperature.

Figure $4 \mathrm{a}$ shows that the lowest resistivity (i.e. lighest carrier concentration) is reached for a slight $\mathrm{Cd}$ overpressure, i.e., for the $\mathrm{Cd} / \mathrm{Te}$ ratio of 1.5. Any deviation from this optimal value results in a significant increase in the resistivity. This result clearly demonstrates that any improper $\mathrm{Cd} / \mathrm{Te}$ pressure ratio during the MBE process results in a considerable reduction of the number of electrically active In atoms which contribute to the free carrier concentration, i.e., it decreases the efficiency of In-doping $[9,10]$.

A strikingly similar dependence on the $\mathrm{Cd} / \mathrm{Te}$ ratio was revealed in a quite different physical phenomenon observed in CdTe:In layers - namely, in the diffusivity of $\mathrm{Mn}$ atoms in the CdTe matrix (see Fig. $4 \mathrm{~b}$ ). For this experiment 2 monolayer thick $\delta$-MnTe layers were embedded in semi-insulating CdTe grown at different $\mathrm{Cd} / \mathrm{Te}$ flux ratios. The samples were thermally annealed in a hydrogen atmosphere at temperatures $330-530^{\circ} \mathrm{C}$. In order to avoid vaporization the samples were placed face-to-face. Redistribution of the $\mathrm{Mn}$ atoms was measured by the SIMS technique. The annealing procedure caused the spreading out of the thin Mn marker in accordance with Fick's law, yielding a Gaussian depth-profile. The diffusivity of Mn atoms was determined from the Arrhenius plot of the half-width of the Gaussian profile [17].

The results presented in Fig. $4 \mathrm{~b}$ can be explained by taking into account the fact that the diffusion coefficient is directly proportional to the concentration of adequate vacancies. For low values of the $\mathrm{Cd} / \mathrm{Te}$ ratio one expects a high concentration of $\mathrm{Cd}$ vacancies and the $\mathrm{Mn}$ diffusion proceeds through the $\mathrm{Cd}$ vacancy-interstitial mechanism. For high $\mathrm{Cd} / \mathrm{Te}$ values, excess of Te vacancies promotes diffusion through $\mathrm{Mn}_{\mathrm{Te}}$ antisite defects. Minimum diffusivity, at $\mathrm{Cd} / \mathrm{Te}=1.5$, corresponds thus to a minimum deviation from the overall stoichiometry. The minimum value of the diffusivity coincides with the observed minimum in the resistivity of the In doped material. This shows that the highest electrical activity is being achieved for the stoichiometric material, and not for the material where a deliberately increased number of $\mathrm{Cd}$ vacancies was to be preferentially occupied by In atoms.

The relation of $\mathrm{Cd}$ to $\mathrm{Te}$ fluxes during the $\mathrm{MBE}$ growth also plays a very important role in the formation of deep defects in CdTe layers. This effect appears to be quite dramatic, as shown in Fig. 5. In layers grown at nearly-stoichiometric flux conditions, i.e. for $1.2<\mathrm{Cd} / \mathrm{Te}<1.8$ one observes a single, very strong DLTS peak. It was established that this peak is attributed to defects whose concentration is closely related to the number of In dopants introduced into the material [11]. In addition, by measurements of the effect of hydrostatic pressure on electron emission and capture rates, it was shown that these defects exhibit properties very similar to those observed for the well-known DX centers in GaAs $[12,18]$.

As in the case of GaAs, the DX-like defect in CdTe is resonant with the conduction band, which was established by Hall effect measurements under hydrostatic pressure. These measurements revealed a level located at about $125 \mathrm{meV}$ above the bottom of the conduction band $[19,20]$. In principle, energy states which 


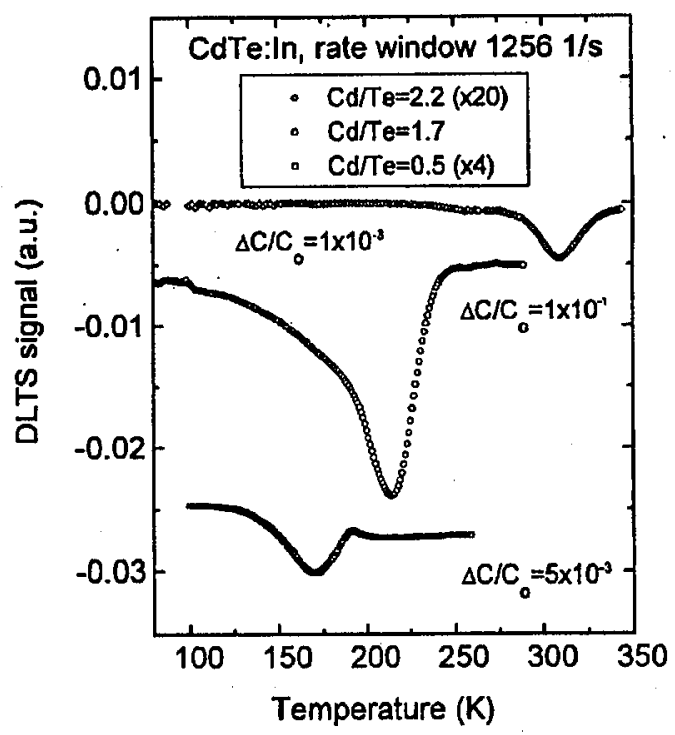

Fig. 5. Typical DLTS spectra of In-doped CdTe films grown at different Cd/Te flux ratios. The spectra were taken at the same rate window of $12561 / \mathrm{s}$ (after Ref. [18]).

are resonant with the conduction band should not be observed in the DLTS experiments. It is because they are not able to bind a carrier for a period of time longer than a millisecond, i.e. a time scale probed by DLTS. However, when electron capturing by such a resonant state is accompanied by the large lattice relaxation, the thermal emission of the carrier takes much more time, because the defect has to absorb many phonons in order to overcome the local lattice distortion.

On the basis of the correlation of the concentration of DX-like defects with the concentration of In dopants can be presumably expected that the DX-like centers in CdTe:In are due to substitutional-interstitial transitions of isolated In atoms, in close analogy to the case of GaAs-based materials. This hypothesis is strongly confirmed by measurements of the defect concentration as a function of the amplitude of bias pulses filling the defects. Because the defect is resonant with the conduction band, its concentration determined by DLTS at reverse bias can be significantly underestimated. It was observed that the forward bias pulses strongly enhance the DLTS signal, as shown in Fig. 6. As a consequence, the measured defect concentration approaches the concentration of In donors introduced into the layers [18].

The DX-like defect has not been seen in layers grown either in excess of Cd or Te, as shown in Fig. 5. Instead, in samples grown at nonstoichiometric conditions DLTS detected two other defect states, but with much lower concentrations which suggests their native origin. The fact that the DX-like states are observed only in layers grown at stoichiometric conditions is very important, because it again points out the isolated In impurity as the microscopic origin of this defect. In particular, it rules out the possibility that the DX-like states are due to pairing of indium 


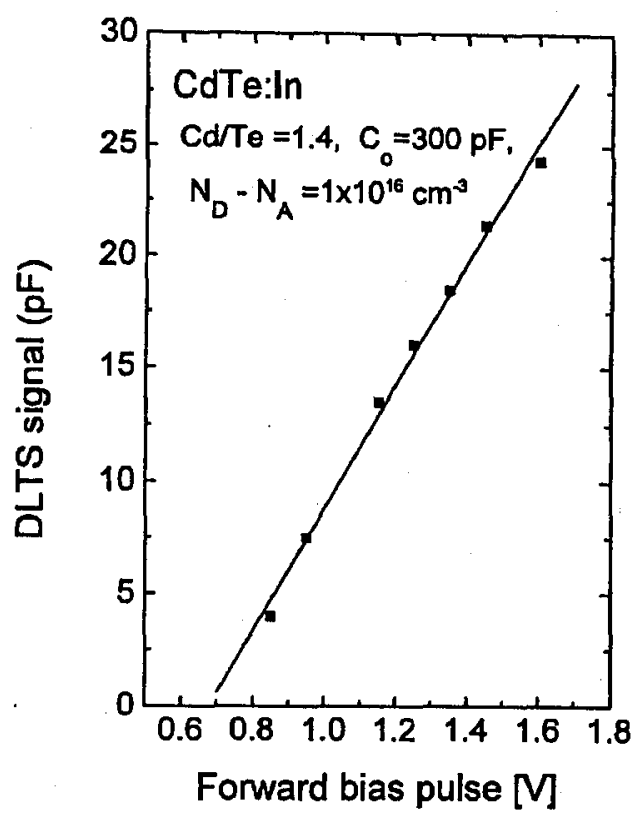

Fig. 6. Dependence of the DLTS signal as a function of the forward bias pulse (after Ref. [18]).

donors with neighboring vacancies. Such a direct observation of the microscopic nature of the DX centers was never reported for GaAs.

\section{Summary}

In this paper we tried to give a short review of the efforts to dope and characterize wide-band gap II-VI materials performed at the University of Notre Dame and at the Institute of Physics in Warsaw. The results clearly show that the effectiveness of the doping, and the properties of the doped materials can be significantly influenced not only by the selection of the dopant species but also by adjusting the stoichiometry of the doped layers.

\section{Acknowledgments}

It is a pleasure to acknowledge our valuable collaboration in the subject of this review with J.K. Furdyna, J. Kossut, M. Dobrowolska, B. Hu, H. Lou, N. Samarth, A.K. Zakrzewski, L. Dobaczewski, A. Barcz, and J. Jaroszyński.

\section{References}

[1] M.A. Haase, J. Qiu, J.M. DePuydt, H. Cheng, Appl. Phys. Lett. 59, 1272 (1991).

[2] II. Jeon, J. Ding, W. Patterson, A.V. Nurmikko, W. Xie, D.C. Grillo, M. Kobayashi, R.L. Gunshor, Appl. Phys. Lett. 59, 3619 (1991).

[3] K. Ohkawa, T. Mitsuyu, O. Yamazaki, J. Appl. Phys. 62, 3216 (1987).

[4] R.M. Park, M.B. Troffer, C.M. Rouleau, J.M. DePuydt, M.A. Haase, Appl. Phys. Lett. 57, 2127 (1990). 
[5] B. Hu, G. Karczewski, II. Luo, N. Samarth, J.K. Furdyna, Phys. Rev. B 47, 9641 (1993).

[6] G. Karczewski, B. Hu, A. Yin, H. Luo, J.K. Furdyna, J. Appl. Phys. 75, 7382 (1994).

[7] B. Hu, G. Karczewski, H. Luo, U. Bindlay, J.K. Furdyna, J. Appl. Phys. 77, 2026 (1995).

[8] G. Karczewski, B. Hu, A. Yin, H. Luo, M. Dobrowolska, J.K. Furdyna, Acta Phys. Pol. A 87, 245 (1995).

[9] G. Karczewski, A.K. Zakrzewski, M. Kutrowski, J. Jaroszyński, W. Dobrowolski, E. Grodzicka, E. Janik, T. Wojtowicz, J. Kossut, Acta Phys. Pol. A 87, 241 (1995).

[10] G. Karczewski, A.K. Zakrzewski, L. Dobaczewski, W. Dobrowolski, E. Grodzicka, J. Jaroszyniski, T. Wojtowicz, J. Kossut, Thin Solid Films 267, 79 (1995).

[11] A.K. Zakrzewski, L. Dobaczewski, G. Karczewski, T. Wojtowicz, J. Kossut, Acta Phys. Pol. A 88, 961 (1995).

[12] A.K. Zakrzewski, L. Dobaczewski, T. Wojtowicz, J. Kossut, G. Karczewski, Proc. 23rd ICPS, Berlin 1996, to be published.

[13] S.M. Shibli, M.C. Tamargo, J.L. DeMiguel, B.J. Skromme, R.E. Nahory, II.II. Farell, J. Appl. Phys. 66, 4295 (1989).

[14] J.M. DePuydt, H. Cheng, J.E. Potts, T.L. Smith, S.K. Mahapatra, J. Appl. Phys. 62, 4756 (1987).

[15] J. Ren, K.A. Bowers, S. Hwang, J.W. Cook, Jr., J.F. Schetzina, J. Crystal Growth 111, 772 (1991).

[16] A. Kamata, T. Uemoto, K. Hirahara, T. Beppu, J. Appl. Plıys. 65, 2561 (1989).

[17] A. Barcz, G. Karczewski, T. Wojtowicz, J. Kossut, J. Cryst. Growth 159, 980 (1996).

[18] A.K. Zakrzewski, L. Dobaczewski, T. Wojtowicz, J. Kossut, G. Karczewski, 23rd ICPS, Berlin 1996, to be published.

[19] D. Wasik, J. Przybytek, M. Baj, G. Karczewski, T. Wojtowicz, A. Zakrzewski, J. Kossut, Mater. Sci. Forum 182-184, 247 (1995).

[20] D. Wasik, J. Przybytek, M. Baj, G. Karczewski, T. Wojtowicz, J. Kossut, J. Cryst. Growth 159, 392 (1996).

[21] A. Barcz, G. Karczewski, private communication. 